\title{
An Improved Car-Following Model in Vehicle Networking Based on Network Control
}

\author{
D. Y. Kong ${ }^{1}$ and H. Y. Xu' ${ }^{2}$ \\ ${ }^{1}$ School of Automotive Studies, Tongji University, Shanghai 201804, China \\ ${ }^{2}$ Sino-German College, Tongji University, Shanghai 201804, China \\ Correspondence should be addressed to H. Y. Xu; xuhaiyang630@163.com
}

Received 24 December 2013; Accepted 19 February 2014; Published 24 March 2014

Academic Editor: Huaicheng Yan

Copyright ( 2014 D. Y. Kong and H. Y. Xu. This is an open access article distributed under the Creative Commons Attribution License, which permits unrestricted use, distribution, and reproduction in any medium, provided the original work is properly cited.

\begin{abstract}
Vehicle networking is a system to realize information interoperability between vehicles and people, vehicles and roads, vehicles and vehicles, and cars and transport facilities, through the network information exchange, in order to achieve the effective monitoring of the vehicle and traffic flow. Realizing information interoperability between vehicles and vehicles, which can affect the traffic flow, is an important application of network control system (NCS). In this paper, a car-following model using vehicle networking theory is established, based on network control principle. The car-following model, which is an improvement of the traditional traffic model, describes the traffic in vehicle networking condition. The impact that vehicle networking has on the traffic flow is quantitatively assessed in a particular scene of one-way, no lane changing highway. The examples show that the capacity of the road is effectively enhanced by using vehicle networking.
\end{abstract}

\section{Introduction}

Network control system (NCS) is a closed-loop feedback control system through real-time network control [1-3]. NCS simplifies cabling system structure and improves the reliability of the system. It has successfully been applied in remote robot, which attracted the attention of many experts. Meanwhile, NCS has become a hotspot of international academic research and obtained a profound impact on the deep-sea robot operation, automobile manufacturing, automotive networking, aerospace industry, and so forth [47]. Vehicle networking is a system to realize information interoperability between vehicles and people, vehicles and roads, vehicles and vehicles, and cars and transport facilities, through the network information exchange, in order to achieve the effective monitoring of the vehicle and traffic flow. Realizing information interoperability between vehicles and vehicles, which can improve road capacity effectively and affect the traffic flow, is an important application of NCS.

In the domestic auto market, the Telematics system has been used in many commercial areas, such as Onstar from GM, Gbook from Toyota, InkaNet from SAIC Motor [8].
These systems may not be perfect, but they basically have the following features: voice navigation system of whole journey, safety services, security services, remote diagnostics, information services, and multimedia entertainment services [9]. These systems play an important role in the emergency information announcement, real-time traffic monitoring, avoidance of traffic accidents, and other aspects [10]. These vehicle systems focus primarily on the information exchange between the car and the call center and have not realized the real-time interoperability communication between vehicles and vehicles, vehicles and road, and vehicles and infrastructure; therefore they are not in the true sense of vehicle networking. The essence of vehicle networking is actually to improve traffic efficiency and avoid accidents through aided driving, automatic collaboration driving, traffic information collection, and so forth [10]. In addition, vehicle networking can also save fuel and reduce carbon dioxide emissions, because there would be no need for drivers to wait for the traffic light under vehicle networking [11]. However, the related researches on vehicle networking are only in its infancy in China, staying in the small-scale closed-loop system. Although some achievements have been achieved, 
there are many thorny issues still need to be resolved to reach the target of establishing a large-scale open-loop cross domain application system [12].

As the core technology of vehicle networking, VANET provides an efficient platform for information sharing and technical support and guarantees for the intelligent transportation systems and the large-scale implementation of vehicle networking [13]. With the development of communication technology in recent years, the research about VANET has also been deepening. But few quantitative studies have been done regarding the impact of vehicle networking on traffic capacity. Moreover, the applications of vehicle networking will significantly change the traffic situation and result in the inapplicability of the traditional car-following model.

In this paper, recent developments of network communication and control technology in the vehicle networking field are summarized, the car-following model based on network control is established, and the impact of vehicle networking on traffic capacity is assessed by quantitative study.

\section{Network Communication and Control Technology in the Vehicle Networking Field}

In recent years, communications protocols, control mechanisms, algorithms, and dynamic VANET technology have obtained certain research results in network communications of vehicle networking [14-17]. The application of the advanced communications technology will make the traffic environment safer, more efficient, and more intelligent.

In standards development and system testing platform fields, IEEE 802.11p promulgated in July 2010 formally, formed the basic framework of the vehicle wireless communication protocols. IEEE 802.11p protocol is the promotion and expansion of IEEE 802.11 in the vehicle networking field and uses IEEE1609 series of protocols as the upper layer protocol. It mainly stipulates the standard of media access control layer (MAC) and physical layer (PHY) in IEEE 802.11p [12]. During that period, more than one test platform was built to validate and test the relative theory, such as Wireless MESH Network Test of the University of Illinois Urbana Champaign Bench, System Test Platform of UCLA, and WAVE System Channel Test Platform of the University of Michigan [18-20].

At the present stage, the research hotspot of VANET is centralized in the following parts based on WAVE protocol (IEEE 802.11p): the application of multichannel coordination in vehicle communication; multicast routing management; and the switching of fixed relay technology and the resource scheduling based on WiMAX protocol (IEEE 802.16). The application of WiMAX technology in vehicle communication networking, which means provide vehicle-mounted broadband wireless access for vehicle and the users inside by using technology based on WiMAX, can overcome some certain weakness of 802.11 protocol, such as small scale coverage, switching the roadside units frequently during the movement of the vehicle, the weakness of quality of service (QOS) support $[21,22]$. So the IEEE 802.16 standards development groups want to use the vehicle-mounted MRS site which provides broadband wireless access service for user terminal groups in the car [23].

To ensure the safety application of QOS (Quality of Service), [24] proposes that multichannel media access control (MAC) mechanism can be used, which means the communication can be achieved using different channels between nodes, so as to get better network throughput volume and delay characteristics than single channel. Location-based multicast routing will also appear, because the original agreement of routing mechanism in WAVE protocol is not entirely suitable to dynamic changing topology of vehicle-mounted communication network. In these routing protocols, the delay correspondingly increases with the increasing in the number of communication hops and speed of vehicle speed $[25,26]$. In [27], to improve multicast routing mechanism, vehicle networking is organized into multiple peer units (cluster), thereby improving scalability mobile environment.

The information communication, that is between vehicles and infrastructure when in high-speed, is becoming another research hotspot. The scheduling mechanism based on two-stage fixed relay can improve system throughput, reduce packet loss rate, and reduce delay time [28]. Besides, some scholars proposed the multihop cellular relay auxiliary switching technology and the group switching technology based on MRS, to guarantee channel QoS indicators and to reduce switching blocking rate and switching delay [29-31]. These theories provide solutions to information exchange process with road side infrastructure for vehicles traveling at high speed.

Currently, there are some research findings on dynamic self-organization,most of which centralize in self-organizing network routing algorithm technology. But the limitation is that most of them remain in the small-scale closed-loop system and do not consider building demand networking with the change of demand and environmental resiliency under the large-scale network environment, which can meet the current task demand [12].

Reference [32], based on the characteristics and research status of VANET analysis, proposes the multidimensional theoretical models and network architecture of VANET; discusses the focal and difficult points of physical layer and related standards, MAC layer and network layer protocol design; explains the broadcast protocol design ideas. Reference [33] proposes a new dynamic distribution perceptual data dissemination system based on Publish-Subscribe for VANET and proposes Notify the Token Mechanism based on the mobility of vehicles. Only token holders can broadcast information to around subscribers. In addition, other scholars have proposed a variety of algorithms to achieve the effective operation of VANET, such as the structure of the basic topology of distributed algorithms, clusterbased routing algorithm method, content-based routing, and dissemination of technology new network architecture [3436]. Reference [37] suggests that percolation theory can be used to analyze the VANET connection. The study found that there is a quantitative relationship between the vehicle density and transmission distance in the network connection. When the vehicle density or transmission range is large enough, there is a jump of a network connection. This result has 
important implications for the development of real-world VANET: this theorem can be used to calculate the minimum transmission range of a reliable network connection to the certain density of vehicles given the vehicle density. Reference [38] constructs a dynamic traffic information system by using a variety of algorithms, such as traffic information collection and traffic incident detection synergy algorithm based on VANET, distributed organization and real-time processing methods of large-scale mass traffic information, locationbased traffic information distribution protocol, and so forth, which can realize the improvement of event detection accuracy and instantaneity in traffic information system and promotion large-scale real-time traffic information processing. Reference [39] introduces the history, characteristics, and application areas of VANET, discusses the advantages and disadvantages of various wireless communication technologies for VANET using analysis and comparison methods. Reference [40] summarizes the current model of simulating vehicle networking simulation including Flow Model of Motorway (FMM), Manhattan Flow Model (MMM), and the random waypoint mobility model and builds a random waypoint model and evaluates its effectiveness through software. Reference [41] classifies the existing VANET simulator and describes and compares several typical emulators.

\section{Establishment of Vehicle Networking Car-Following Model}

In the circumstance that the vehicle networking has been built perfectly, that is, VANET has been completed constructed, real-time communication between vehicles and vehicles can be achieved. In the transportation system, one car can transmit driving parameter packages to the surrounding vehicles at high frequency. The surrounding vehicles can receive the date packages at a high reception rate and low delay time and then get the driving state of the car, so that the surrounding vehicles can take appropriate measures. Through the above steps, it is possible to drive unmanned, thus effectively reducing distance between vehicles, reducing traffic accidents, and improving transportation efficiency.

In the context, the traditional classical transport theory will not be all applicable to vehicle networking system as the applicable conditions change. The classical car-following model needs to be improved so as to describe the transportation state based on vehicle networking.

3.1. Basic Assumption. In the model, the following basic assumptions are made.

(1) Vehicle automatic mode. In the fleet, all vehicles take autopilot mode. That is, when the information is received from the leading vehicle, following vehicles can automatically take appropriate measures. Thus, the reaction time of human can be ignored. This is easy to achieve in the background of vehicle networking.

(2) Information transmission without delay. When the leading vehicle takes appropriate measures, it can simultaneously transmit information to surrounding vehicles, and then other vehicles can receive information and immediately take appropriate measures. When the transmission frequency and the reception frequency are sufficiently high and the delay time is short enough, we can assume the delay time can be negligible in the model. In addition, in a small-scale fleet, that is to say there are a small number of vehicles, the time lag that the last car and the leading car take corresponding measures is less enough.

(3) Acceleration consistency. All vehicles in fleet have the same maximum acceleration in braking and driving process.

3.2. Analysis about Driving Characteristics. In the vehicle networking, when vehicles have been compiled as fleet, vehicles are still in the nonfree-running state, but driving characteristics differ from the traditional classical theories. The driving characteristics of a car-following model in vehicle networking are as follows.

(1) Restriction. In fleet, back cars closely follow the front cars, meeting "immediately followed" requirement. Meanwhile, the speed of back cars cannot be greater than the cars in front, only swing near the front cars in fleet. As the automotive control is electronic and accurate, swing amplitude is minimal and difference in speed is negligible, so the speed is quite suitable. This is the "speed conditions." "Immediately followed" requirement, the equal speed and small distance constitute the restriction of the car-following model in vehicle networking.

(2) Without hysteretic. The restriction characteristic above shows that the changes of driving condition of front vehicle lead to the changes of back vehicle. In vehicle networking condition, based on the assumption that vehicles take autopilot mode and no delay in information transmission, the driving condition of front and back vehicles changes to the same extent simultaneously, which means no reaction time.

(3) Transitivity. Similar to the traditional model, the condition of the entire fleet will change when the driving condition of the leading car changes. The driving condition of 1 st vehicle restricts the 2 nd one; the 2 nd one restricts the 3 rd one and so on. Because there is no delay of delivery, the information is passed back along the fleet smoothly.

3.3. Description and Derivation. In vehicle networking, assuming the distance between leading vehicle and following vehicle in the car-following model is $s(t)$, the information delay time is $T$. Delay time $T$ is calculated from the moment leading car brake to the moment following car taking brake measure. The speed of following car stays invariability in $T$. The relative position of two vehicles at the moment of $t$ is shown in Figure 1. In Figure 1, $n$ represents the leading car, and $n+1$ represents the following car. At the moment of $t$, 


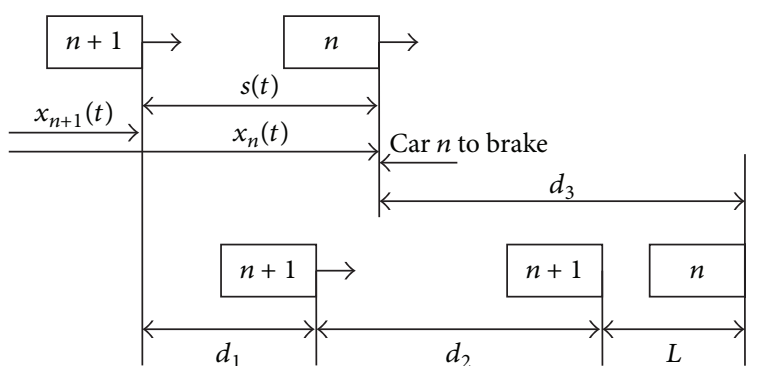

FIgURE 1: The relative position of two adjacent vehicles.

the leading car starts to break; the relative position of the two vehicles at some time is shown in the lower half of Figure 1.

In this figure,

$L$ is the distance between head of two vehicles;

$d_{1}$ is the distance vehicle $n+1$ traveled by the reaction time $\Delta t$;

$d_{2}$ is the distance vehicle $n+1$ traveled from brake;

$d_{3}$ is the distance vehicle $n$ traveled from brake.

Thus, at time $t$, the required distance of the front vehicle that suddenly stops without collision is

$$
s(t)=x_{n}(t)-x_{n+1}(t)=d_{1}+d_{2}+L-d_{3} .
$$

The speed of the vehicle is

$$
v(t)=\frac{d x(t)}{d t}=\dot{x}(t) .
$$

The acceleration is

$$
a(t)=\frac{d^{2} x(t)}{d t^{2}}=\ddot{x}(t) .
$$

Since $\dot{x}(t)$ is a finite and under the assumption that $T$ approaches 0 then $d_{1} \approx 0$ :

$$
s(t)=\frac{\dot{x}_{n+1}^{2}(t)}{2 \ddot{x}_{n+1}(t)}+L-\frac{\dot{x}_{n}^{2}(t)}{2 \ddot{x}_{n}(t)} .
$$

As vehicles of the fleet have taken autopilot mode, the speed of each vehicle is same. The back vehicle will take brake with the same acceleration, when receiving the brake signal from front vehicle. Then

$$
\dot{x}_{n+1}(t)=\dot{x}_{n}(t) \quad \ddot{x}_{n+1}(t)=\ddot{x}_{n}(t) .
$$

So

$$
s(t)=L .
$$

From the above calculation, we can see that the distance between front and back vehicles remain unchanged during the braking; that is, the distance is the same in travelling and after braking. Therefore, in the vehicle networking context, if all vehicles have the capabilities of communicating with others, the distance between leading and following vehicles can be infinitely small in car-following process.
TABLE 1: The main variables for the model application.

\begin{tabular}{ll}
\hline Variable & Meaning \\
\hline$v_{f 1}$ & Ideal free flow speed in traditional conditions \\
$v_{f 2}$ & Ideal free flow speed in vehicle networking conditions \\
$Q_{m 2}$ & $\begin{array}{l}\text { Road capacity in vehicle networking conditions } \\
\text { Average distance between vehicles in single lane, in }\end{array}$ \\
$D_{1}$ & $\begin{array}{l}\text { traditional conditions, when reaching the blocking } \\
\text { density. Unit: m }\end{array}$ \\
& $\begin{array}{l}\text { Average distance between vehicles in single lane, in } \\
D_{2}\end{array}$ \\
& $\begin{array}{l}\text { vehicle networking conditions, when reaching the } \\
\text { blocking density. Preceding analysis shows } D_{2} \approx 0\end{array}$ \\
$K_{j 1}$ & $\begin{array}{l}\text { Average length of car (m) } \\
\text { Traffic density maximum in traditional conditions, }\end{array}$ \\
$K_{j 2}$ & $\begin{array}{l}\text { block density } \\
\text { blocking density }\end{array}$ \\
$Q_{m 1}$ & Road capacity in traditional conditions
\end{tabular}

\section{Model Application}

This improved car-following model can be used to calculate the typical traffic scene road traffic capacity, thus assessing the influence of the vehicle networking to the traffic capacity in quantitative assessment.

Assuming that the vehicle fleet forms one-way traffic on a flat straight highway without considering overtaking, lane change, toll stations, and so forth, this paper uses traditional and improved linear car-following models, respectively, and analyzes two different scene traffic capacities. The main parameters are shown in Table 1.

4.1. In Traditional Conditions. In traditional situation, the equation to describe the relationship between traffic flow and density is as follows:

$$
Q=v_{f}\left(K-\frac{K^{2}}{K_{j}}\right) .
$$

So, when $d Q / d K=0, Q$ gets the maximum, we can get

$$
Q_{m}=\frac{1}{4} v_{f} K_{j}
$$

As we know $K_{j 1}=1000 /\left(L+D_{1}\right)$.

So the road capacity can be describe as

$$
Q_{m 1}=\frac{250}{L+D_{1}} v_{f 1}
$$

4.2. In Vehicle Networking Conditions. In vehicle networking conditions $Q=K v$.

$K$ and $v$ have no necessary connection, so

$$
Q_{m 2}=v_{f 2} K_{j 2}
$$

because of

$$
K_{\mathrm{j} 2}=\frac{1000}{L+D_{2}} \approx \frac{1000}{L}
$$


So we can get

$$
Q_{m 2}=\frac{1000}{L} v_{f 2}
$$

4.3. Impact Assessment. In conclusion, the effect of car networking on traffic capacity is

$$
K=\frac{Q_{m 2}}{Q_{m 1}}=4 \frac{L+D_{1}}{L} \cdot \frac{v_{f 2}}{v_{f 1}} .
$$

On account of $v_{f 2} \geq v_{f 1}$, there is

$$
K \geq 4 \cdot \frac{L+D_{1}}{L} .
$$

From the analysis above, we can see that in the ideal state of no lane change highway, the role of vehicle networking will make the traffic capacity of roads improve $4 \cdot\left(\left(L+D_{1}\right) / L\right)$ times compared to the original.

On the highway, it is assumed that the distance between the high-speed cars is not less than $100 \mathrm{~m}$, and the average car length is about $5 \mathrm{~m}$. In an ideal condition, compared with the traditional conditions, vehicle networking will increase the maximum traffic flow of highway by more than 84 times and correspondingly improve the traffic capacity of road greatly.

This situation is mainly thanks to the following two reasons.

(a) Shortening of vehicle distance. In vehicle networking condition, vehicles can establish a real-time communication and exchange information instantaneously. That allows vehicles to take the same measures almost at the same time without reaction time. The distance between vehicles can be shortened without loss in safety.

(b) No direct relevance between speed and density. In the traditional conditions, velocity decreases with the increase of density. But in vehicle networking conditions, speed and density have no direct relationship. When the density is very high, the fleet can still run at the speed of free stream.

\section{Conclusion}

The core technology in realizing the information exchange of vehicle networking is the communication technology represented by network control technology. The development level of communication technology affects VANET application directly. A car-following model using vehicle networking theory is established, describing the traffic condition in vehicle networking condition. The impact that vehicle networking has on the traffic flow is quantitatively assessed in the particular scene of one-way, no lane changing highway. The examples show that the capacity of the road is effectively enhanced by using vehicle networking.

This paper provides a method to assess the impact of vehicle networking on the traffic flow quantitatively. But the amendment of the model in complex conditions, such as lane changing and steering, remains to be further studied.

\section{Conflict of Interests}

The authors declare that there is no conflict of interests regarding the publication of this paper.

\section{Acknowledgment}

The authors would like to acknowledge the support from The National Key Technology R\&D Program of the Ministry of Science and Technology of China (Grant no. 2013BAG06B04).

\section{References}

[1] W. Zhang, M. S. Branicky, and S. M. Phillips, "Stability of networked control systems," IEEE Control Systems Magazine, vol. 21, no. 1, pp. 84-97, 2001.

[2] G. C. Walsh, H. Ye, and L. Bushnell, "Stability analysis of networked control systems," in Proceedings of the American Control Conference (ACC '99), pp. 2876-2880, Institute of Electrical and Electronics Engineers Inc, San Diego, Cailf, USA, June 1999.

[3] G. C. Walsh and H. Ye, "Scheduling of networked control systems," IEEE Control Systems Magazine, vol. 21, no. 1, pp. 5765, 2001.

[4] C. Rhodes, M. Morari, L. S. Tsimring, and N. F. Rulkov, "Data-based control trajectory planning for nonlinear systems," Physical Review E, vol. 56, no. 3, pp. 2398-2406, 1997.

[5] H. Zhang, H. Yan, F. Yang, and Q. Chen, "Quantized control design for impulsive fuzzy networked systems," IEEE Transactions on Fuzzy Systems, vol. 19, no. 6, pp. 1153-1162, 2011.

[6] M. Arif, T. Ishihara, and H. Inooka, "Incorporation of experience in iterative learning controllers using locally weighted learning," Automatica, vol. 37, no. 6, pp. 881-888, 2001.

[7] L. X. Zhang, H. J. Gao, and O. Kaynak, "Network-induced constraints in networked control systems-a survey," IEEE Transactions on Industrial Informatics, vol. 9, no. 1, pp. 403-416, 2013.

[8] W. W. Hu, "The internet revolution of vehicles," Business Value, 2013.

[9] J. Deng, L. P. Xia, L. H. Lu, and Z. W. Zhang, "Technical feasibility analysis of vehicle network in independent brand automobile," Automobile Parts, no. 2, pp. 56-58, 2012.

[10] G. Cheng and D. Guo, "The current status and development of vehicle networking," Mobile Communications, no. 17, pp. 23-26, 2011.

[11] T. Tielert, M. Killat, H. Hartenstein, R. Luz, S. Hausberger, and T. Benz, "The impact of traffic-light-to-vehicle communication on fuel consumption and emissions," in Proceedings of the 2nd International Internet of Things Conference (IOT '10), December 2010.

[12] J. J. Cheng and M. C. Zhou, "The third important information space: vehicle networking technology and application analysis," Overseas Scholars, 2012.

[13] C. Chen, Research on key issues VANET system security [Ph.D. thesis], Fudan University, 2011.

[14] H. Gao, T. Chen, and J. Lam, "A new delay system approach to network-based control," Automatica, vol. 44, no. 1, pp. 39-52, 2008.

[15] H. Yan, H. Shi, H. Zhang, and F. Yang, "Quantized $H_{\infty}$ control for networked systems with communication constraints," Asian Journal of Control, vol. 15, no. 5, pp. 1468-1476, 2013. 
[16] H. Zhang, H. Yan, T. Liu, and Q. Chen, "Fuzzy controller design for nonlinear impulsive fuzzy systems with time delay," IEEE Transactions on Fuzzy Systems, vol. 19, no. 5, pp. 844-856, 2011.

[17] H. Zhang, Q. Chen, H. Yan, and J. Liu, "Robust $H_{\infty}$ filtering for switched stochastic system with missing measurements," IEEE Transactions on Signal Processing, vol. 57, no. 9, pp. 3466-3474, 2009.

[18] T. Shen, Experiments on a multichannel multi-interface wireless mesh network [M.S. thesis], University of Illinois at UrbanaChampaign, 2008.

[19] G. Pau and A. Rowstron, PVRP: Efficient Disruption Tolerant Discovery in Vehicular Ad Hoc Networks, ACM MobiComm, 2009.

[20] F. Q. Liu and L. H. Shan, "Vehicular heterogeneous networks architecture and key technologies," ZTE Communications, vol. 16, no. 3, pp. 47-51, 2010.

[21] K. Yang, S. Ou, H.-H. Chen, and J. He, "A multihop peercommunication protocol with fairness guarantee for IEEE 802.16-based vehicular networks," IEEE Transactions on Vehicular Technology, vol. 56, no. 6, pp. 3358-3370, 2007.

[22] L. Shan, F. Liu, and K. Yang, "Performance analysis of group handover scheme for IEEE 802. 16j-enabled vehicular networks," in Proceedings of the Joint International Conferences on Asia-Pacific Web Conference and Web-Age Information Management, 2009.

[23] IEEE C802.16j-D5, "Draft amendment for local and metropolitan area networks, Part 16: air interface for mobile broadband wireless systems multihop relay specification," 2008.

[24] J. Crichigno, M.-Y. Wu, and W. Shu, "Protocols and architectures for channel assignment in wireless mesh networks," Ad Hoc Networks, vol. 6, no. 7, pp. 1051-1077, 2008.

[25] C. Lochert, H. Hartenstein, J. Tian, H. Fussler, D. Hermann, and M. Mauve, "A routing strategy for vehicular ad hoc networks in city environments," in Proceedings of the IEEE Intelligent Vehicles Symposium, 2003.

[26] G. Korkmaz, F. Özgüner, E. Ekici, and Ü. Özgüner, "Urban multi-hop broadcast protocol for inter-vehicle communication systems," in Proceedings of the 1st ACM International Workshop on Vehicular Ad Hoc Networks (VANET '04), pp. 76-85, October 2004.

[27] F. Li and Y. Wang, "Routing in vehicular ad hoc networks: a survey," IEEE Vehicular Technology Magazine, vol. 2, no. 2, pp. 12-22, 2007.

[28] L. Wang, Y. Ji, and F. Liu, "Adaptive subframe partitioning and efficient packet scheduling in OFDMA cellular system with fixed decode-and-forward relays," IEICE Transactions on Communications, vol. E92-B, no. 3, pp. 755-765, 2009.

[29] S. Jeon and S. Lee, "A relay-assisted handover technique with network coding over multihop cellular networks," IEEE Communications Letters, vol. 11, no. 3, pp. 252-254, 2007.

[30] H. Yan, Z. Su, H. Zhang, and F. Yang, "Observer-based $H_{\infty}$ control for discrete-time stochastic systems with quantisation and random communication delays," IET Control Theory \& Applications, vol. 7, no. 3, pp. 372-379, 2013.

[31] L. Shan, F. Liu, L. Wang, and Y. Ji, "Predictive group handover scheme with channel borrowing for mobile relay systems," in Proceedings of the IEEE International Wireless Communications and Mobile Computing Conference (IWCMC '08), pp. 153-158, August 2008.

[32] M. O. Cherif, S. M. Senouci, and B. Ducourthial, "Vehicular network self-organizing architectures," in Proceedings of the 5th IEEE GCC Conference and Exhibition (GCC '09), March 2009.
[33] L. Wu, M. Liu, X. Wang, and H. Gong, "Dynamic distributionaware data dissemination for vehicular ad hoc networks," in Proceedings of the 2nd International Conference on Future Computer and Communication (ICFCC '10), pp. V2353-V2360, May 2010.

[34] A. Yair and M. Segal, "Near-optimal, reliable and self-organizing hierarchical topology in VANET," in Proceedings of the 8th ACM International Workshop on Vehicular Inter-Networking, September 2011.

[35] D. Rajini Girinath and S. Selvan, "A novel cluster based routing algorithm for hybrid mobility model in VANET," International Journal of Computer Applications, vol. 1, no. 15, 2010.

[36] G. Arnould, D. Khadraoui, and Z. Habbas, "A self-organizing content centric network model for hybrid vehicular Ad-Hoc networks," in Proceedings of the 1st ACM International Symposium on Design and Analysis of Intelligent Vehicular Networks and Applications (DIVANet '11), pp. 15-22, November 2011.

[37] X. Jin, W. Su, and Y. Wei, "A study of the VANET connectivity by percolation theory," in Proceedings of the IEEE Consumer Communications and Networking Conference (CCNC '11), pp. 85-89, January 2011.

[38] Y. Xiang, "Dynamic traffic data mining based on vehicular ad hoc networking," ZTE Technology Journal, vol. 17, no. 3, pp. 2934, 2011.

[39] C.-Y. Chang, Y. Xiang, and M.-L. Shi, "Development and status of vehicular ad hoc networks," Journal on Communications, vol. 28, no. 11, pp. 116-126, 2007.

[40] V. D. Khairnar and S. N. Pradhan, "Mobility models for Vehicular Ad-hoc Network simulation," in Proceedings of the IEEE Symposium on Computers and Informatics (ISCI '11), pp. 460-465, March 2011.

[41] L. Xiao, R.-F. Li, and J. Luo, "Simulation of vehicular ad hoc networks: a survey," Journal of System Simulation, vol. 21, no. 17, pp. 5330-5356, 2009. 


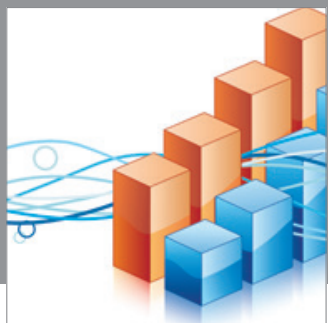

Advances in

Operations Research

mansans

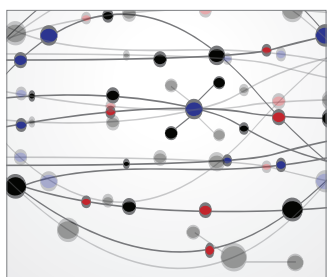

The Scientific World Journal
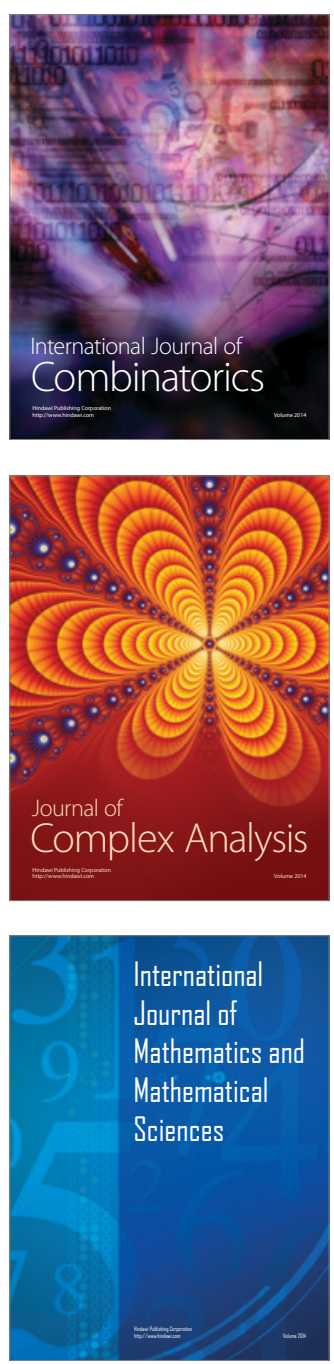
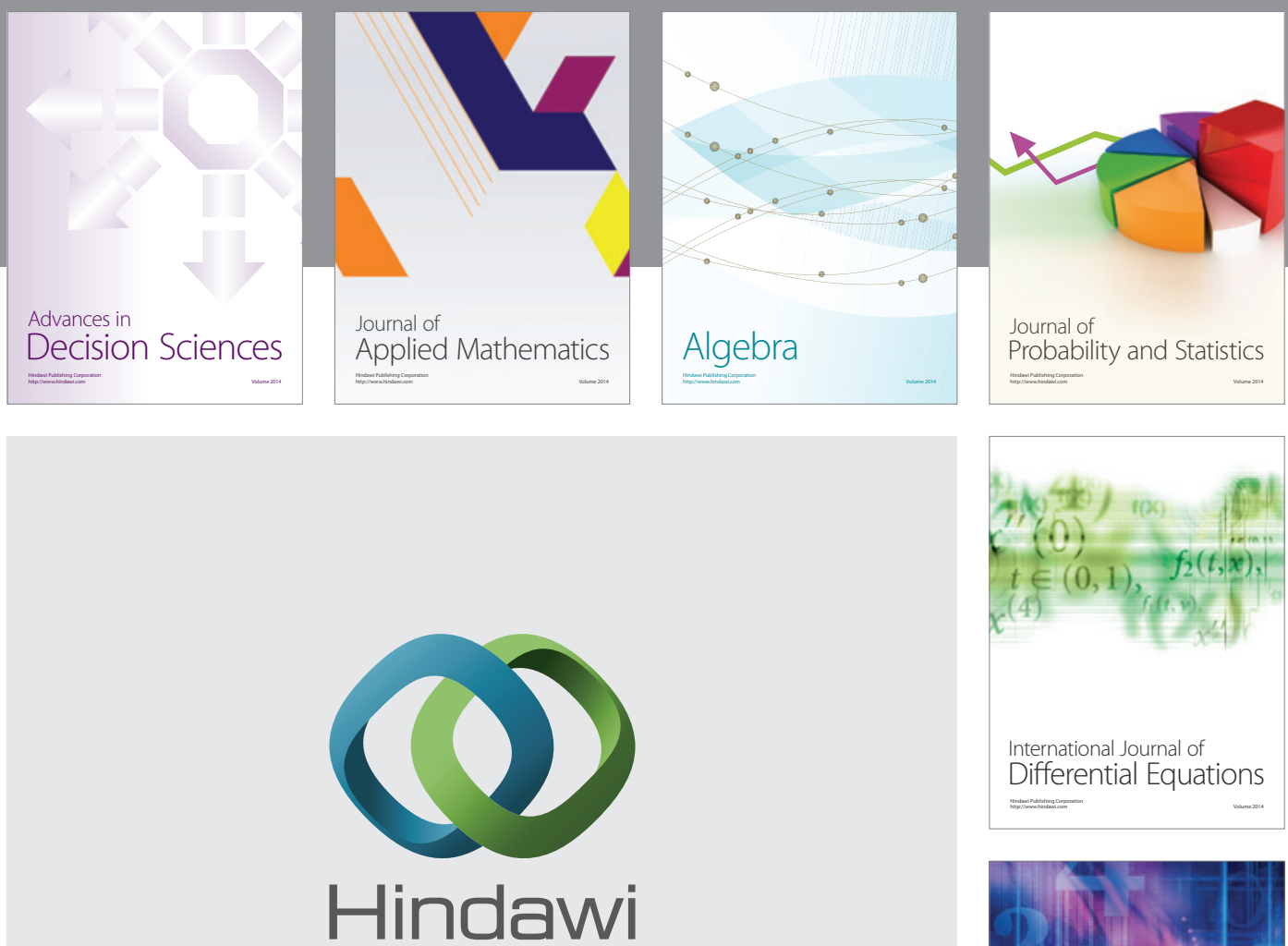

Submit your manuscripts at http://www.hindawi.com
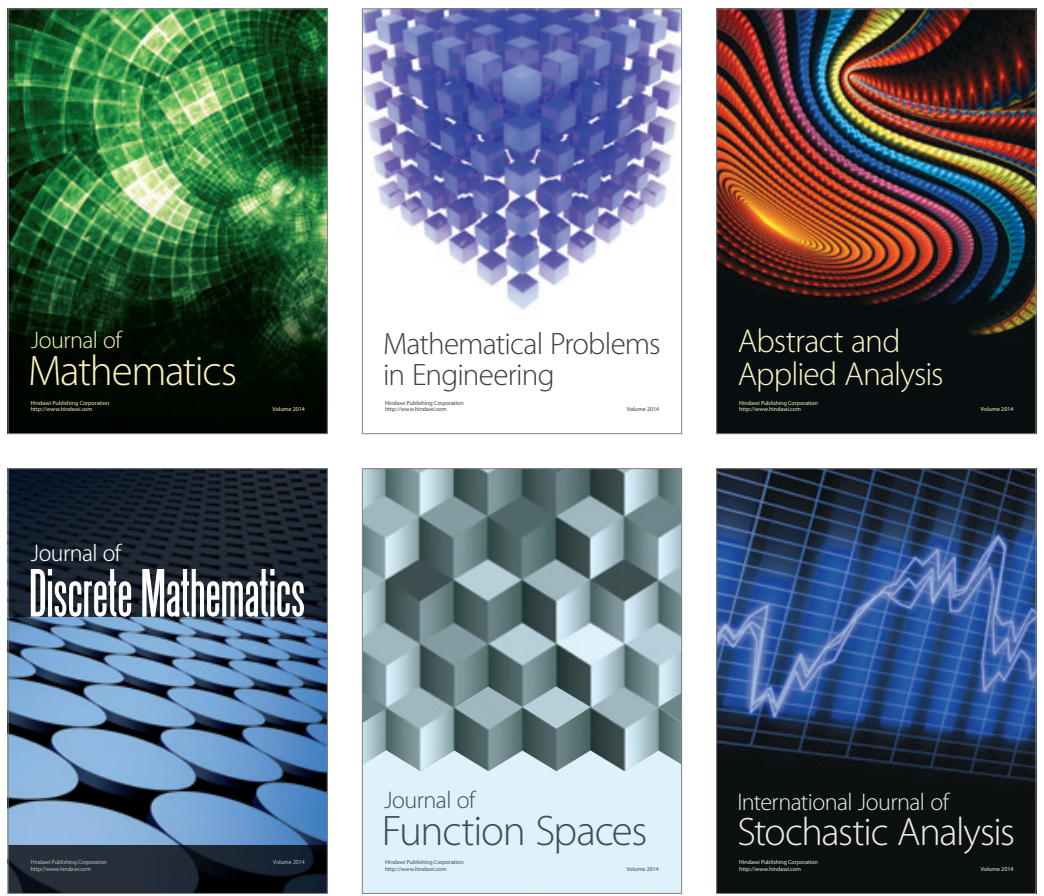

Journal of

Function Spaces

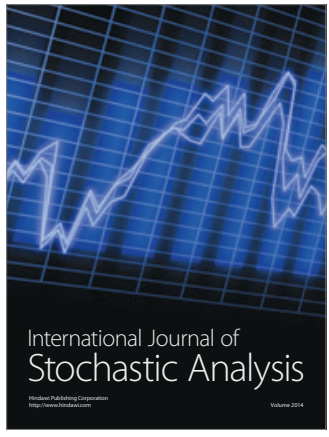

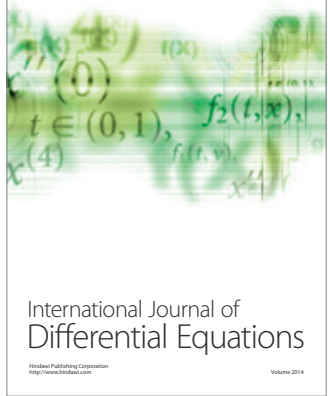
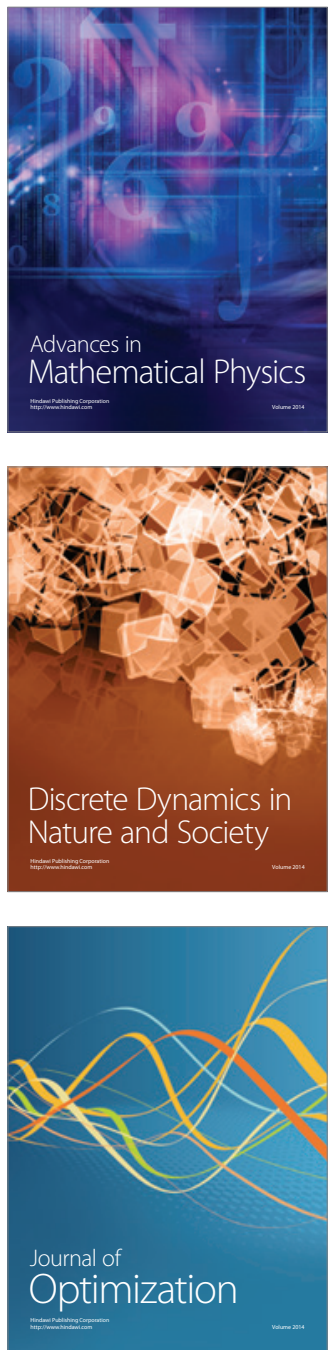